\title{
New Stability Results for Patterns in a Model of Long-Wavelength Convection
}

\author{
Anne C. Skeldon ${ }^{a}$ and Mary Silber ${ }^{b}$ \\ ${ }^{a}$ Department of Mathematics, City University, Northampton Square, London, \\ EC1V OHB UK, England \\ ${ }^{\mathrm{b}}$ Department of Engineering Sciences and Applied Mathematics, Northwestern \\ University, Evanston, IL 60208 USA
}

\begin{abstract}
We consider the transition from a spatially uniform state to a steady, spatiallyperiodic pattern in a partial differential equation describing long-wavelength convection [1]. This both extends existing work on the study of rolls, squares and hexagons and demonstrates how recent generic results for the stability of spatially-periodic patterns may be applied in practice. We find that squares, even if stable to roll perturbations, are often unstable when a wider class of perturbations is considered. We also find scenarios where transitions from hexagons to rectangles can occur. In some cases we find that, near onset, more exotic spatially-periodic planforms are preferred over the usual rolls, squares and hexagons.
\end{abstract}

\section{Introduction}

Pattern forming instabilities arise in a wide number of physical and chemical problems. Model partial differential equations are used to try to capture the essential features of the observed transitions. In many interesting examples such as Rayleigh-Bénard convection and reaction-diffusion problems, the model equations are invariant under all translations, rotations and reflections in the plane and patterns arise at a transition from a trivial solution consisting of no pattern. Linear stability analysis of the trivial solution leads to a critical curve describing how the wavenumber for instability, $k$, depends on a parameter, $\mu$, in the problem. For parameter values below the critical curve the trivial solution is stable. At a critical parameter, $\mu_{c}$, instability onsets at a critical wavenumber $k_{c}$. 
Weakly nonlinear analysis is often used to try to predict the type of patterns observed once the trivial solution becomes unstable. Two aspects make this particularly difficult: firstly, the rotational invariance of the problem means that instability to a single wavenumber gives instability to a whole circle of wavevectors. In other words, if the trivial solution is unstable to rolls then it is unstable to rolls with any orientation in the plane. Secondly, for $\mu>\mu_{c}$, not just a single wavenumber but a whole band of wavenumbers is unstable. Often this second problem is addressed by assuming that, sufficiently close to $\mu_{c}$, boundaries in any real problem will select out one particular wavenumber and modes with neighbouring wavenumbers will be suppressed. In the case of the first problem, a tacit assumption is often made that, for a given wavenumber, only a finite number of critical wavevectors are relevant. For example, four critical wavevectors oriented at $\frac{\pi}{2}$ to each other (see figure $1(\mathrm{a})$ ) are chosen or six critical wavevectors oriented at $\frac{\pi}{3}$ to each other are chosen (see figure $1(\mathrm{~b})$ ). In both cases the critical wavevectors generate a periodic lattice of points, a square lattice in the first case and a hexagonal lattice in the second. Consequently, the circle of critical wavevectors is replaced by a finite set and a finite dimensional centre manifold exists for the problem, of dimension four in the case of squares and of dimension six in the case of hexagons. If critical wavevectors are used which do not generate a periodic lattice then there is no reason a priori why a finite dimensional centre manifold exists, since modes arbitrarily close to critical occur. While non-periodic cases have been considered [2], their validity requires an additional assumption on the suppression of these near critical modes.

Weakly nonlinear analysis using wavevectors on a square lattice or a hexagonal lattice as shown in figure 1, provide a framework for examining the relative stability of either squares and rolls or hexagons and rolls respectively. In both cases generic bifurcation equations have been derived using symmetry arguments [3-5]. A more complete stability analysis for rolls has been performed, for example by Brattkus and Davis [6], who consider the relative stability of two sets of rolls oriented at an arbitrary angle for a problem arising in crystal growth. Similarly, a more complete analysis can be performed for squares and hexagons by considering families of different square and hexagonal lat- 
tices (for specific examples see figure 2). This problem has a high degree of symmetry and using group theoretic arguments, Dionne and Golubitsky show that, for each lattice, additional branches other than hexagons, rolls or squares bifurcate as primary bifurcations $[9,10]$. In spite of the fact that the Fourier transform of the new patterns involves only one critical wavenumber, in physical space they appear to have more than one lengthscale. Such "superlattice" patterns have recently been observed in the Faraday crispation experiment $[7,8]$. (For examples see figures 4 and 8 below.)

Using symmetry arguments, Dionne et al. derive the generic bifurcation equations for the families of square and hexagonal lattices and examine the stability of certain primary bifurcation branches in terms of the coefficients of the bifurcation equations [11]. This stability analysis enables two types of statement to be made. Firstly, since each lattice problem corresponds to a subspace of the original unbounded problem, and since hexagons and squares each exist on a whole family of lattices, the stability of these planforms can be considered to a countably infinite number of perturbations. While this is not equivalent to completely determining the stability of squares and hexagons in an unbounded domain, it does considerably extend previous results. Secondly, each individual lattice corresponds to either a square or hexagonal domain with periodic boundary conditions. For each lattice, the relative stability of the primary branches known to exist from [9] can be calculated. These results, contained in [11], have not as yet been applied to any specific partial differential equation and it is this issue we address here.

In this paper, we re-examine the relative stability of spatially-periodic solutions to a partial differential equation considered by Knobloch [1]. This equation,

$$
\begin{aligned}
f_{t}=\alpha f-\mu \nabla^{2} f-\nabla^{4} f+ & \kappa \nabla \cdot|\nabla f|^{2} \nabla f \\
& +\beta \nabla \cdot \nabla^{2} f \nabla f-\gamma \nabla \cdot f \nabla f+\delta \nabla^{2}|\nabla f|^{2},
\end{aligned}
$$

describes a number of long-wavelength partial differential equations which arise in convection problems. For example, when $\kappa=1, \beta=\delta=\gamma=0$ we recover the planform equation for convection in a layer between two poorly 
conducting boundaries [12], and when $\kappa=1, \gamma=0, \beta=-\frac{\sqrt{ } 7}{8}, \delta=-\frac{3 \sqrt{ } 7}{8}$ equation (1) models long-wavelength Marangoni convection [13]. Further examples are given in [1]. For equation (1) we demonstrate, that with relatively little additional analysis, we can derive all the coefficients necessary to apply the results from [11]. We thereby significantly extend Knobloch's stability results by inclusion of the additional perturbations.

In section 2 we define the critical modes which generate square and hexagonal lattices used here and the resulting generic bifurcation equations. The derivation of the coefficients of the bifurcation equations is given in section 3 . Then in section 4 we discuss the results for two specific cases. In Case I we take $\gamma=0, \kappa=+1$. Knobloch called this Case B, the nature of our conclusions for his Case A are similar and we do not present them in detail. For Case I, provided $\beta \neq \delta$, the coefficient of the quadratic term in the bifurcation equations for the hexagonal lattices is nonzero. This quadratic term renders all of the primary solution branches for the hexagonal lattices unstable at bifurcation [14] and thus we restrict our attention to the square lattice bifurcation problems. We divide our discussion into two parts: in section 4.1.1 we consider the stability of squares and rolls in an unbounded domain by considering their stability on the whole family of square lattices; in section 4.1.2 we consider the particular example of long-wavelength Marangoni convection and show that different bifurcation scenarios can occur for different square lattices. In Case II we consider $\gamma / k^{2}=\delta-\beta, \kappa=+1$. This choice of parameters yields a degenerate bifurcation problem for the hexagonal lattice since the coefficient of the quadratic term in the bifurcation equations is zero. Stable primary branches are therefore a possibility for all lattices and we consider both square and hexagonal types. We first discuss what can be deduced of the stability of rolls, hexagons and squares in an unbounded domain in section 4.2.1; then in section 4.2.2 we discuss the unfolding expected if the coefficient of the quadratic term is non-zero but sufficiently small. Finally, in 4.2 .3 we discuss the specific example of Marangoni convection for different hexagonal lattices. Our conclusions are summarised in section 5 . 


\section{Preliminaries}

In order to apply the analysis given in [11], we consider sets of eight or twelve critical modes whose wavevectors generate square or hexagonal lattices respectively, where the length of the critical wavevector is greater than the distance between neighbouring points on the lattice. A typical example is shown in figure 2(a) for the square lattice. This figure should be contrasted with figure 1(a), which shows the wavevectors which are used conventionally in pattern selection studies. In both figures, a circle representing the critical wavevectors for the original unbounded problem has been superimposed on the lattice. In figure 1(a) this circle only intersects the lattice at four points and there are consequently four critical modes, whereas in figure 2(a) the circle intersects the lattice at eight points and hence there are eight critical modes. Sufficiently close to the critical value of the parameter, $\mu_{c}$, all other modes, represented by vectors not of length $k_{c}$, will be damped. A family of finer and finer lattices can be constructed each with eight points on the critical circle. Each lattice can be encoded by a pair of integers, $(m, n)$; for example, the lattice shown in figure $2(\mathrm{a})$ corresponds to the case $(2,1)$ i.e. $\mathbf{K}_{\mathbf{1}_{\mathbf{s}}}$ is two squares of the lattice across and one up. The eight wavevectors consist of two sets of four wavevectors, $\left( \pm \mathbf{K}_{\mathbf{1}_{\mathbf{s}}}, \pm \mathbf{K}_{\mathbf{2}_{\mathbf{s}}}\right)$ and $\left( \pm \mathbf{K}_{\mathbf{3}_{\mathbf{s}}}, \pm \mathbf{K}_{\mathbf{4}_{\mathbf{s}}}\right)$, that comprise squares and are rotated by an angle $\theta_{s}$ relative to each other. An alternative way to specify each lattice is therefore through the lattice angle $\theta_{s}$, where,

$$
\theta_{s}=\cos ^{-1}\left(\frac{2 m n}{m^{2}+n^{2}}\right)
$$

and $m>n>0$ are relatively prime positive integers that are not both odd. Reducing the circle of critical wavevectors to four critical wavevectors, as shown in figure 1(a), is equivalent to changing the original unbounded domain to a box whose side length is $\frac{1}{k_{c}}$ and applying periodic boundary conditions. Using eight critical wavevectors, illustrated in figure $2($ a) for the case $(m, n)=$

$(2,1)$, corresponds to changing the domain to a box of side length $\frac{\sqrt{m^{2}+n^{2}}}{k_{c}}$ and again applying periodic boundary conditions. 
In a similar way, a family of hexagonal lattices exists where the number of critical wavevectors is twelve. These twelve wavevectors consist of two sets of six wavevectors, $\left( \pm \mathbf{K}_{\mathbf{1}_{\mathbf{h}}}, \pm \mathbf{K}_{\mathbf{2}_{\mathbf{h}}}, \pm \mathbf{K}_{\mathbf{3}_{\mathbf{h}}}\right)$ and $\left( \pm \mathbf{K}_{\mathbf{4}_{\mathbf{h}}}, \pm \mathbf{K}_{\mathbf{5}_{\mathbf{h}}}, \pm \mathbf{K}_{\mathbf{6}_{\mathbf{h}}}\right)$, that comprise hexagons rotated at the angle $\theta_{h}$ given by

$$
\theta_{h}=\cos ^{-1}\left(\frac{m^{2}+2 m n-2 n^{2}}{2\left(m^{2}-m n+n^{2}\right)}\right)
$$

where now $m>n>\frac{m}{2}$ are relatively prime positive integers, and where $m+n$ is not a multiple of 3 . An example of the case $(m, n)=(3,2)$ is shown in figure 2(b). For both the square and hexagonal lattices the requirement that $m$ and $n$ are positive integers ensures that the critical wavevectors generate a periodic lattice. This is necessary if the centre manifold theorem is to be invoked to formally justify the use of finite-dimensional bifurcation equations. The remaining conditions on $m$ and $n$ ensure that each lattice angle corresponds to a genuinely different case [10].

For the square case, letting $z_{j}$ be the complex amplitude of mode $e^{i\left(\mathbf{K}_{j_{s}} \cdot \mathbf{r}\right)}$ where $\mathbf{r}=(x, y)$ then the generic bifurcation equations take the form

$$
\begin{aligned}
& \dot{z}_{1}=\lambda z_{1}+\left(a_{1}\left|z_{1}\right|^{2}+a_{2}\left|z_{2}\right|^{2}+a_{3}\left|z_{3}\right|^{2}+a_{4}\left|z_{4}\right|^{2}\right) z_{1}+O\left(|\mathbf{z}|^{5}\right), \\
& \dot{z}_{2}=\lambda z_{2}+\left(a_{2}\left|z_{1}\right|^{2}+a_{1}\left|z_{2}\right|^{2}+a_{4}\left|z_{3}\right|^{2}+a_{3}\left|z_{4}\right|^{2}\right) z_{1}+O\left(|\mathbf{z}|^{5}\right), \\
& \dot{z}_{3}=\lambda z_{3}+\left(a_{3}\left|z_{1}\right|^{2}+a_{4}\left|z_{2}\right|^{2}+a_{1}\left|z_{3}\right|^{2}+a_{2}\left|z_{4}\right|^{2}\right) z_{1}+O\left(|\mathbf{z}|^{5}\right), \\
& \dot{z}_{4}=\lambda z_{4}+\left(a_{4}\left|z_{1}\right|^{2}+a_{3}\left|z_{2}\right|^{2}+a_{2}\left|z_{3}\right|^{2}+a_{1}\left|z_{4}\right|^{2}\right) z_{1}+O\left(|\mathbf{z}|^{5}\right) .
\end{aligned}
$$

One recovers the bifurcation equations associated with the wavevectors given in figure 1 by restricting to the subspace $z_{3}=z_{4}=0$. Equations (4) have six known types of primary branch which are listed in table 1 along with their stability assignments in terms of the coefficients. Note that rolls and squares are the same on all lattices, but the rhombs (rectangles), super squares and anti-squares take a different form depending on $(m, n)$. For example, changing $(m, n)$ changes the aspect ratio of the rhombs. An example of one of the super square solutions can be seen in figure 4. Further examples of the different planforms are given in $[9-11]$.

For the hexagonal lattice problem, letting $z_{j}$ be the complex amplitude of mode $e^{i\left(\mathbf{K}_{j_{h}} \cdot \mathbf{r}\right)}$ then the generic bifurcation equations take the form 
Table 1

Signs of eigenvalues for primary bifurcation branches on the square lattice; $a_{1}, \ldots, a_{4}$ are coefficients in the bifurcation equation (4).

\begin{tabular}{|l|l|}
\hline Planform & Signs of non-zero eigenvalues \\
\hline Rolls $(\mathrm{R})$ & $\operatorname{sgn}\left(a_{1}\right), \quad \operatorname{sgn}\left(a_{2}-a_{1}\right), \quad \operatorname{sgn}\left(a_{3}-a_{1}\right), \quad \operatorname{sgn}\left(a_{4}-a_{1}\right)$ \\
\hline $\mathbf{z}=A_{R}(1,0,0,0)$ & $\operatorname{sgn}\left(a_{1}+a_{2}\right), \quad \operatorname{sgn}\left(a_{1}-a_{2}\right), \quad \operatorname{sgn}\left(a_{3}+a_{4}-a_{1}-a_{2}\right)$ \\
$\mathbf{z}=A_{S}(1,1,0,0)$ & \\
\hline Rhombs $\left(\operatorname{Rh}_{s 1, m, n}\right)$ & $\operatorname{sgn}\left(a_{1}+a_{3}\right), \quad \operatorname{sgn}\left(a_{1}-a_{3}\right), \quad \operatorname{sgn}\left(a_{2}+a_{4}-a_{1}-a_{3}\right)$ \\
$\mathbf{z}=A_{R h}\left(\theta_{s}\right)(1,0,1,0)$ & \\
\hline Rhombs $\left(\operatorname{Rh}_{s 2, m, n}\right)$ & $\operatorname{sgn}\left(a_{1}+a_{4}\right), \quad \operatorname{sgn}\left(a_{1}-a_{4}\right), \quad \operatorname{sgn}\left(a_{2}+a_{3}-a_{1}-a_{4}\right)$ \\
$\mathbf{z}=A_{R h}\left(\theta_{s}+\frac{\pi}{2}\right)(1,0,0,1)$ & $\operatorname{sgn}\left(a_{1}+a_{2}+a_{3}+a_{4}\right), \quad \operatorname{sgn}\left(a_{1}+a_{2}-a_{3}-a_{4}\right)$ \\
\hline Super Squares $\left(\mathrm{SS}_{m, n}\right)$ & $\operatorname{sgn}\left(a_{1}-a_{2}+a_{3}-a_{4}\right), \quad \operatorname{sgn}\left(a_{1}-a_{2}-a_{3}+a_{4}\right)$ \\
$\mathbf{z}=A_{S S}(1,1,1,1)$ & $\operatorname{sgn}\left(\mu_{0}\right)$, where $\mu_{0}=O\left(A_{S S}^{2(m+n-1)}\right)$ \\
\hline Anti-Squares $\left(\mathrm{AS}_{m, n}\right)$ & $\operatorname{same}$ as super squares, except $\mu_{0} \rightarrow-\mu_{0}$ \\
$\mathbf{z}=A_{A S}(1,1,-1,-1)$ & \\
\hline
\end{tabular}

$$
\begin{aligned}
\dot{z}_{1}= & \lambda z_{1}+\epsilon \bar{z}_{2} \bar{z}_{3} \\
& +\left(b_{1}\left|z_{1}\right|^{2}+b_{2}\left|z_{2}\right|^{2}+b_{2}\left|z_{3}\right|^{2}+b_{4}\left|z_{4}\right|^{2}+b_{5}\left|z_{5}\right|^{2}+b_{6}\left|z_{6}\right|^{2}\right) z_{1}+O\left(|\mathbf{z}|^{4}\right), \\
\dot{z}_{2}= & \lambda z_{2}+\epsilon \bar{z}_{3} \bar{z}_{1} \\
& +\left(b_{2}\left|z_{1}\right|^{2}+b_{1}\left|z_{2}\right|^{2}+b_{2}\left|z_{3}\right|^{2}+b_{6}\left|z_{4}\right|^{2}+b_{4}\left|z_{5}\right|^{2}+b_{5}\left|z_{6}\right|^{2}\right) z_{2}+O\left(|\mathbf{z}|^{4}\right), \\
\dot{z}_{3}= & \lambda z_{3}+\epsilon \bar{z}_{1} \bar{z}_{2} \\
& +\left(b_{2}\left|z_{1}\right|^{2}+b_{2}\left|z_{2}\right|^{2}+b_{1}\left|z_{3}\right|^{2}+b_{5}\left|z_{4}\right|^{2}+b_{6}\left|z_{5}\right|^{2}+b_{4}\left|z_{6}\right|^{2}\right) z_{3}+O\left(|\mathbf{z}|^{4}\right), \\
\dot{z}_{4}= & \lambda z_{4}+\epsilon \bar{z}_{6} \bar{z}_{5} \\
& +\left(b_{4}\left|z_{1}\right|^{2}+b_{5}\left|z_{2}\right|^{2}+b_{6}\left|z_{3}\right|^{2}+b_{1}\left|z_{4}\right|^{2}+b_{2}\left|z_{5}\right|^{2}+b_{2}\left|z_{6}\right|^{2}\right) z_{4}+O\left(|\mathbf{z}|^{4}\right), \\
\dot{z}_{5}= & \lambda z_{5}+\epsilon \bar{z}_{4} \bar{z}_{6} \\
& +\left(b_{5}\left|z_{1}\right|^{2}+b_{4}\left|z_{2}\right|^{2}+b_{6}\left|z_{3}\right|^{2}+b_{2}\left|z_{4}\right|^{2}+b_{1}\left|z_{5}\right|^{2}+b_{2}\left|z_{6}\right|^{2}\right) z_{5}+O\left(|\mathbf{z}|^{4}\right), \\
\dot{z}_{6}= & \lambda z_{6}+\epsilon \bar{z}_{5} \bar{z}_{4} \\
& +\left(b_{6}\left|z_{1}\right|^{2}+b_{5}\left|z_{2}\right|^{2}+b_{4}\left|z_{3}\right|^{2}+b_{2}\left|z_{4}\right|^{2}+b_{2}\left|z_{5}\right|^{2}+b_{1}\left|z_{6}\right|^{2}\right) z_{6}+O\left(|\mathbf{z}|^{4}\right) .
\end{aligned}
$$

The standard hexagonal bifurcation problem is recovered by restricting to the subspace $z_{4}=z_{5}=z_{6}=0$. Primary branches for equations (5) are listed in table 2. Note that rolls and hexagons are the same on all lattices, but the rhombs and the super hexagons take a different form depending on $(m, n)$. Examples of one of the rhombs and one of the super hexagon states are given in figure $6(\mathrm{~b})$ and figure 8 respectively. As for the square lattice, 
further examples of the different planforms may be seen in [9-11].

\section{Calculation of the Coefficients}

The form of the bifurcation equations (4) and (5) is determined by the symmetry of the problem. However, the coefficients, and therefore the stability of the different planforms, depend upon the particular application. One important result in [11] is that, although high order terms in the bifurcation equations are required to find the relative stability of some planforms, much is fixed by the cubic order truncation. Therefore, in this section we determine the coefficients of the quadratic and cubic terms in the bifurcation equations (4) and (5) from the long-wavelength equation (1). Our approach is to infer these quantities from the branching equations for rolls, simple squares, rhombs, and simple hexagons, which are computed using perturbation theory below . The reason we take this indirect approach is twofold. Firstly, it is simpler than a direct approach since although it requires four separate calculations, each is only onedimensional: the direct approach requires one eight-dimensional calculation and one twelve-dimensional calculation. Secondly, for cases such as this one, where stability on the simplest square and hexagonal lattices has been considered already, we wish to emphasize that only one further one-dimensional calculation is required to obtain all the information for the extended stability analysis presented here.

We let

$$
\begin{aligned}
& f=\epsilon f_{0}+\epsilon^{2} f_{1}+\epsilon^{3} f_{2}+\ldots \\
& \mu=\mu_{c}+\epsilon \mu_{1}+\epsilon \mu_{2}+\ldots,
\end{aligned}
$$

where $\mu$ is the bifurcation parameter and instability onsets at the critical value $\mu_{c}=2(-\alpha)^{1 / 2}$, at the critical wavenumber $k_{c}=(-\alpha)^{1 / 2}$. In turn, we take

$$
\begin{aligned}
\text { Rolls : } & f_{0}=A_{R} e^{i k x}+\cdots+\text { c.c. } \\
\text { Squares : } & f_{0}=A_{S}\left(e^{i k x}+e^{i k y}\right)+\cdots+\text { c.c. } \\
\text { Hexagons : } & f_{0}=A_{H}\left(e^{i k x}+e^{i k(-x+\sqrt{3} y) / 2}+e^{i k(-x-\sqrt{3} y) / 2}\right)+\cdots+c . c . \\
\text { Rhombs : } & f_{0}=A_{R h}\left(e^{i k x}+e^{i k(c x+s y)}\right)+\cdots+\text { c.c., where } c \equiv \cos (\theta), s \equiv \sin (\theta) .
\end{aligned}
$$


Table 2

Branching equations and signs of eigenvalues for primary bifurcation branches on the hexagonal lattice; $\epsilon, b_{1}, \ldots, b_{6}$ are coefficients in the bifurcation equation (5).

\begin{tabular}{|c|c|}
\hline Planform and branching equation & Signs of non-zero eigenvalues \\
\hline $\begin{array}{l}\text { Rolls }(\mathrm{R}) \\
\mathbf{z}=A_{R}(1,0,0,0,0,0) \\
0=\lambda A_{R}+b_{1} A_{R}^{3}+O\left(A_{R}^{5}\right)\end{array}$ & $\begin{array}{l}\operatorname{sgn}\left(b_{1}\right), \quad \operatorname{sgn}\left(\epsilon A_{R}+\left(b_{2}-b_{1}\right) A_{R}^{2}\right), \\
\operatorname{sgn}\left(-\epsilon A_{R}+\left(b_{2}-b_{1}\right) A_{R}^{2}\right), \\
\operatorname{sgn}\left(b_{4}-b_{1}\right), \quad \operatorname{sgn}\left(b_{5}-b_{1}\right), \quad \operatorname{sgn}\left(b_{6}-b_{1}\right) .\end{array}$ \\
\hline 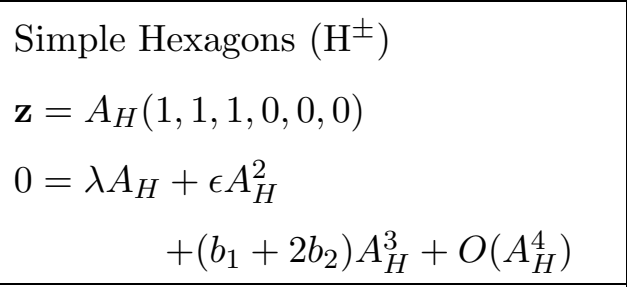 & $\begin{array}{l}\operatorname{sgn}\left(\epsilon A_{H}+2\left(b_{1}+2 b_{2}\right) A_{H}^{2}\right) \\
\operatorname{sgn}\left(-\epsilon A_{H}+\left(b_{1}-b_{2}\right) A_{H}^{2}\right) \\
\operatorname{sgn}\left(-\epsilon A_{H}+\left(b_{4}+b_{5}+b_{6}-b_{1}-2 b_{2}\right) A_{H}^{2}\right) \\
\operatorname{sgn}\left(-\epsilon A_{H}+O\left(A_{H}^{3}\right)\right)\end{array}$ \\
\hline $\begin{array}{l}\text { Rhombs }\left(\operatorname{Rh}_{h 1, m, n}\right) \\
\mathbf{z}=A_{R h}\left(\theta_{h}\right)(1,0,0,1,0,0) \\
0=\lambda A_{R h}+\left(b_{1}+b_{4}\right) A_{R h}^{3}+O\left(A_{R h}^{5}\right)\end{array}$ & $\begin{array}{l}\operatorname{sgn}\left(b_{1}+b_{4}\right), \quad \operatorname{sgn}\left(b_{1}-b_{4}\right) \\
\operatorname{sgn}\left(\mu_{1}\right), \quad \operatorname{sgn}\left(\mu_{2}\right), \quad \text { where, } \\
\mu_{1}+\mu_{2}=\left(-2 b_{1}-2 b_{4}+2 b_{2}+b_{5}+b_{6}\right) A_{R h}^{2}, \\
\mu_{1} \mu_{2}=-\epsilon^{2} A_{R h}^{2}+\left(b_{1}+b_{4}-b_{2}-b_{5}\right)\left(b_{1}+b_{4}-b_{2}-b_{6}\right) A_{R h}^{4} .\end{array}$ \\
\hline $\begin{array}{l}\text { Rhombs }\left(\operatorname{Rh}_{h 2, m, n}\right) \\
\mathbf{z}=A_{R h}\left(\theta_{h}+\frac{2 \pi}{3}\right)(1,0,0,0,1,0) \\
0=\lambda A_{R h}+\left(b_{1}+b_{5}\right) A_{R h}^{3}+O\left(A_{R h}^{5}\right)\end{array}$ & $\begin{array}{l}\operatorname{sgn}\left(b_{1}+b_{5}\right), \quad \operatorname{sgn}\left(b_{1}-b_{5}\right) \\
\operatorname{sgn}\left(\mu_{1}\right), \quad \operatorname{sgn}\left(\mu_{2}\right), \quad \text { where, } \\
\mu_{1}+\mu_{2}=\left(-2 b_{1}-2 b_{5}+2 b_{2}+b_{4}+b_{6}\right) A_{R h}^{2}, \\
\mu_{1} \mu_{2}=-\epsilon^{2} A_{R h}^{2}+\left(b_{1}+b_{5}-b_{2}-b_{4}\right)\left(b_{1}+b_{5}-b_{2}-b_{6}\right) A_{R h}^{4} .\end{array}$ \\
\hline $\begin{array}{l}\text { Rhombs }\left(\mathrm{Rh}_{h 3, m, n}\right) \\
\mathbf{z}=A_{R h}\left(\theta_{h}-\frac{2 \pi}{3}\right)(1,0,0,0,0,1) \\
0=\lambda A_{R h}+\left(b_{1}+b_{6}\right) A_{R h}^{3}+O\left(A_{R h}^{5}\right)\end{array}$ & $\begin{array}{l}\operatorname{sgn}\left(b_{1}+b_{6}\right), \quad \operatorname{sgn}\left(b_{1}-b_{6}\right) \\
\operatorname{sgn}\left(\mu_{1}\right), \quad \operatorname{sgn}\left(\mu_{2}\right), \quad \text { where, } \\
\mu_{1}+\mu_{2}=\left(-2 b_{1}-2 b_{6}+2 b_{2}+b_{4}+b_{5}\right) A_{R h}^{2}, \\
\mu_{1} \mu_{2}=-\epsilon^{2} A_{R h}^{2}+\left(b_{1}+b_{6}-b_{2}-b_{4}\right)\left(b_{1}+b_{6}-b_{2}-b_{5}\right) A_{R h}^{4},\end{array}$ \\
\hline $\begin{array}{l}\text { Super Hexagons }\left(\mathrm{SH}_{m, n}^{ \pm}\right)^{* *} \\
\begin{aligned} \mathbf{z} & =A_{S H}(1,1,1,1,1,1) \\
0 & =\lambda A_{S H}+\epsilon A_{S H}^{2}+\left(b_{1}+2 b_{2}\right) A_{S H}^{3} \\
& +\left(b_{4}+b_{5}+b_{6}\right) A_{S H}^{3}+O\left(A_{S H}^{4}\right)\end{aligned}\end{array}$ & $\begin{array}{l}\operatorname{sgn}\left(\epsilon A_{S H}+2\left(b_{1}+2 b_{2}+b_{4}+b_{5}+b_{6}\right) A_{S H}^{2}\right) \\
\operatorname{sgn}\left(\epsilon A_{S H}+2\left(b_{1}+2 b_{2}-b_{4}-b_{5}-b_{6}\right) A_{S H}^{2}\right) \\
\operatorname{sgn}\left(-\epsilon A_{S H}+O\left(A_{S H}^{3}\right)\right), \operatorname{sgn}\left(-\epsilon A_{S H}+O\left(A_{S H}^{3}\right)\right)^{*} \\
\operatorname{sgn}\left(\mu_{1}\right), \quad \operatorname{sgn}\left(\mu_{2}\right), \quad \text { where, } \\
\mu_{1}+\mu_{2}=-4 \epsilon A_{S H}+4\left(b_{1}-b_{2}\right) A_{S H}^{2}, \\
\mu_{1} \mu_{2}=4\left(\epsilon A_{S H}-\left(b_{1}-b_{2}\right) A_{S H}^{2}\right)^{2} \\
\left.\quad-2\left(\left(b_{4}-b_{5}\right)^{2}+\left(b_{4}-b_{6}\right)^{2}+\left(b_{5}-b_{6}\right)^{2}\right)\right) A_{S H}^{4} \\
\operatorname{sgn}\left(\mu_{0}\right), \text { where, } \mu_{0}=O\left(A_{S H}^{2(m-1)}\right) .\end{array}$ \\
\hline
\end{tabular}

* These two eigenvalues differ at $O\left(A_{S H}^{3}\right)$.

** Super triangles [8] have the same eigenvalues except $\operatorname{sgn}\left(\mu_{0}\right) \rightarrow-\operatorname{sgn}\left(\mu_{0}\right)$. 
At $O\left(\epsilon^{3}\right)$ the solvability condition gives an equation for the amplitudes $A_{R}, A_{S}, A_{H}, A_{R h}$ respectively. That is,

$$
\begin{aligned}
\dot{A}_{R} & =\lambda A_{R}+C_{R} A_{R}^{3}+O\left(A_{R}^{5}\right), \\
\dot{A}_{S} & =\lambda A_{S}+C_{S} A_{S}^{3}+O\left(A_{S}^{5}\right), \\
\dot{A}_{H} & =\lambda A_{H}+\epsilon A_{H}^{2}+C_{H} A_{H}^{3}+O\left(A_{H}^{4}\right), \\
\dot{A}_{R h} & =\lambda A_{R h}+C_{R h} A_{R h}^{3}+O\left(A_{R h}^{5}\right),
\end{aligned}
$$

where $\lambda=k^{2}\left(\mu-\mu_{c}\right)$ and

$$
\begin{aligned}
\epsilon & =k^{4}\left(\beta+\frac{\gamma}{k^{2}}-\delta\right) \\
C_{R} & =-k^{4}\left(3 \kappa+2\left(\beta+\delta-\frac{\epsilon}{3 k^{4}}\right)\left(\delta+\frac{\epsilon}{3 k^{4}}\right)\right) \\
C_{S} & =C_{R}-2 k^{4}\left(\kappa+2\left(\delta^{2}-\frac{\epsilon^{2}}{k^{8}}\right)\right) \\
C_{H} & =C_{R}-\frac{3 k^{4}}{2}\left(4 \kappa+\left(\beta+2 \delta-\frac{\epsilon}{k^{4}}\right)\left(2 \delta+\frac{\epsilon}{k^{4}}\right)\right) \\
C_{R h}(\theta) & =C_{S}-4 k^{4} \cos ^{2} \theta\left(\kappa+\beta \delta-\frac{\beta \epsilon}{k^{4}\left(1-4 \cos ^{2} \theta\right)}-\frac{8 \epsilon^{2}\left(1-2 \cos ^{2} \theta\right)}{k^{8}\left(1-4 \cos ^{2} \theta\right)^{2}}\right) .
\end{aligned}
$$

Note that, as expected, $C_{R h}\left(\frac{\pi}{2}\right)=C_{S}$. Also note that $C_{R h}$ diverges as $\theta \rightarrow \frac{\pi}{3}$ since when $\theta=\frac{\pi}{3}$ there is resonance between $e^{i k x}$ and $e^{i k(c x+s y)}$.

The cubic coefficients in the equivariant bifurcation equations (4) and (5) are readily expressed in terms of the branching coefficients $C_{R}, C_{S}, C_{H}$ and $C_{R h}$. For instance, if (4) is restricted to the simple squares subspace $\mathbf{z}=$ $\left(A_{S}, A_{S}, 0,0\right)$, we find

$$
\dot{A}_{S}=\lambda A_{S}+\left(a_{1}+a_{2}\right) A_{S}^{3}
$$

Comparing equation (9) with the appropriate branching equation from equations (7) gives $a_{1}+a_{2}=C_{S}$. Similarly, by restricting to subspaces for rolls and rhombs for the square lattice problems (4), we find

$$
\begin{aligned}
& a_{1}=C_{R}, \quad a_{2}=C_{S}-C_{R}, \\
& a_{3}=C_{R h}\left(\theta_{s}\right)-C_{R}, \quad a_{4}=C_{R h}\left(\theta_{s}+\frac{\pi}{2}\right)-C_{R},
\end{aligned}
$$


where $\theta_{s} \in\left(0, \frac{\pi}{2}\right)$ takes on one of the discrete set of values (2). Similarly, restriction to subspaces for rolls, hexagons and rhombs for the hexagonal lattice bifurcation problems (5) leads to

$$
\begin{aligned}
& b_{1}=C_{R}, \quad b_{2}=\frac{1}{2}\left(C_{H}-C_{R}\right), \quad b_{4}=C_{R h}\left(\theta_{h}\right)-C_{R}, \\
& b_{5}=C_{R h}\left(\theta_{h}+\frac{2 \pi}{3}\right)-C_{R}, \quad b_{6}=C_{R h}\left(\theta_{h}-\frac{2 \pi}{3}\right)-C_{R},
\end{aligned}
$$

where $\theta_{h} \in\left(0, \frac{\pi}{3}\right)$ takes on one of the values in the discrete set (3).

Note that the expressions for $C_{R}, C_{S}, \epsilon$, and $C_{H}$ are given in [1] and, in that paper, are used to calculate the coefficients $a_{1}, a_{2}, b_{1}$ and $b_{2}$. The remaining expression for $C_{R h}$ is the only additional calculation required to enable all the remaining coefficients in the bifurcation equations (4) and (5) to be found.

\section{Stability Results}

In this section we use the bifurcation equations (4) and (5) to determine the relative stability of the steady planforms which are given in section 2 . The results depend on the parameters $\kappa, \beta, \delta$, and $\gamma$ in the long-wave equation (1). They also depend on the size of the periodic domain through the lattice parameters $(m, n)$. We restrict our discussion to two cases and, where possible, compare and contrast our results with those given in [1].

The relative stability of each primary branch is obtained by linearising about the solutions of the bifurcation problem, (4) or (5), and then explicitly calculating the eigenvalues. These calculations were carried out in [11] and the signs of the eigenvalues are summarized in tables 1 and 2 for the square and hexagonal lattices, respectively. The sign of the first quantity listed for each planform gives the branching direction; if this eigenvalue is negative (positive), then the branch is supercritical (subcritical). If $\epsilon \neq 0$ then simple hexagons and super hexagons bifurcate transcritically; all other patterns arise through pitchfork bifurcations. We distinguish between the two branches of hexagons, denoted $\mathrm{H}^{+}$and $\mathrm{H}^{-}$, which satisfy $A_{H}>0$ and $A_{H}<0$, respectively. Similarly, there are two distinct branches of super hexagons, denoted by $\mathrm{SH}_{m, n}^{ \pm}$. 
Omitted from the tables are the zero eigenvalues associated with translations of the patterns, and also information about the multiplicities of each eigenvalue; this information can be found in [11]. Note that certain eigenvalues in tables 1 and 2 are not determined at cubic order in the bifurcation equations. For instance, the relative stability of super squares and anti-squares depends on a resonant term of $O\left(|\mathbf{z}|^{2 m+2 n-1}\right)$. However, in this case, if super squares and anti-squares are neutrally stable at cubic order, then, generically, exactly one of the two states is stable. There is an analogous stability result for super hexagons and triangles [8].

In the case of the square lattice, the eigenvalues that depend only on $a_{1}$ and $a_{2}$ can be determined by considering the restricted bifurcation problem $z_{3}=z_{4}=$ 0 in equation (4). Similarly, those results for the hexagonal lattice that depend only on $\epsilon, b_{1}$ and $b_{2}$ can be obtained by considering the simpler hexagonal bifurcation problem. In general, the signs of all the remaining eigenvalues are dependent on the choice of lattice. However, in the special case when $\epsilon=0$, we find that those eigenvalues which are unchanged on permutation of $a_{3}$ and $a_{4}$ are independent of $\theta_{s}$, and those which are unchanged on permutation of $b_{4}, b_{5}$ and $b_{6}$ are independent of $\theta_{h}$. This is due to the particularly simple $\theta$ dependence of $C_{R h}(\theta)$ in equation (8) when $\epsilon=0$. Also, in the case of the square lattices, results for angles close to $\frac{\pi}{6}$ and $\frac{\pi}{3}$ must be interpreted with care because of the singularity in $C_{R h}(\theta)$ at $\theta=\frac{\pi}{3}$ that occurs due to resonant interactions.

In each of the cases we discuss below, we evaluate the signs of the eigenvalues for each planfrom and determine if and where they change sign. We present the results in the form of bifurcation sets separating different regions of stability and instability for the relevant patterns. Note that along the stability boundaries themselves, the bifurcation problem is degenerate and the bifurcation equations (4) and (5) are insufficient to locally determine the bifurcation structure. Some of these degenerate points have been analysed by Knobloch [1]. 


\subsection{Case I: $\gamma=0, \kappa=+1$}

In case I, solutions on the hexagonal lattices are unstable at onset unless $\beta=\delta$ i.e. $\epsilon=0$ in equations (5). Thus here we focus on the square lattice problems and defer discussion of the hexagonal cases to section 4.2.

\subsubsection{The stability of squares and rolls}

If we can show that squares or rolls are unstable on any one lattice then they must be unstable in the original unbounded problem sufficiently close to onset. From the hexagonal lattice result, we can immediately infer that rolls are unstable to hexagonal perturbations for $\beta \neq \delta$. Here we show that squares are also unstable unless $\beta$ is sufficiently close to $\delta$. The bifurcation sets for rolls and for squares in the $(\beta, \delta)$-plane are presented in figures $3(\mathrm{a})$ and $(\mathrm{b})$ respectively.

The sign of the branching eigenvalue for rolls, $\operatorname{sgn}\left(a_{1}\right)$, is always negative, so rolls always bifurcate supercritically. The sign of $a_{2}-a_{1}$ determines the relative stability of squares and rolls and the corresponding bifurcation line, $a_{1}=a_{2}$ in figure $3(\mathrm{a})$, is identical to the line $q(0)=0$ given in figure 2(b) of [1]. The relative stability of rolls and the two rhombic patterns is determined by the signs of $a_{3}-a_{1}$ and $a_{4}-a_{1}$. Since $a_{3}$ and $a_{4}$ are dependent on $\theta_{s}$, the precise position of the corresponding bifurcation curves $a_{1}=a_{3}$ and $a_{1}=a_{4}$ depends on the choice of lattice: those shown in figure 3(a) are for the case $(m, n)=(2,1)$. Qualitatively the picture is the same in all cases corresponding to $0<\theta_{s}<\frac{\pi}{4}$. For $\theta_{s}>\frac{\pi}{4}$, the picture is similar except $a_{3}-a_{1}$ and $a_{4}-a_{1}$ switch roles. The region of stability is indicated by the shaded wedges in the $(\beta, \gamma)$-plane between $a_{1}=a_{2}$ and $a_{1}=a_{4}\left(a_{1}=a_{2}\right.$ and $a_{1}=a_{3}$ if $\left.\theta_{s}>\frac{\pi}{4}\right)$. As the lattice angle, $\theta_{s}$, approaches $\frac{\pi}{6}$ or $\frac{\pi}{3}$ the region of stability of the rolls is reduced to a narrower and narrower region occurring only for large $|\beta|$ and $|\delta|$. At precisely $\theta=\frac{\pi}{3}$ (or the complementary $\frac{\pi}{6}$ ) the hexagonal lattice must be considered.

In figure 3(b) we show the analogous bifurcation set for squares showing where each of the three expressions given in table 1 for the eigenvalues for squares 
change sign. The lines $a_{1}=a_{2}$ and $a_{1}=-a_{2}$ correspond to the lines $q(0)=0$ and $p_{N}(0)=0$ given by Knobloch. In his study he found the squares were preferred to rolls for the region between these two curves. However, this region is significantly reduced when instability to super square (or anti-square) states is included through the eigenvalue $a_{3}+a_{4}-a_{1}-a_{2}$. The position of the corresponding bifurcation line given by $a_{3}+a_{4}=a_{1}+a_{2}$ is again dependent on the value of the lattice angle. As the lattice angle approaches $\frac{\pi}{6}$ or $\frac{\pi}{3}$ the region of stability of the squares is reduced to a narrower and narrower region. Interestingly this narrow region always includes the line $\beta=\delta$, for which the hexagonal problem is degenerate. Recall that, for hexagonal lattices, it is only in this degenerate case that stable planforms can exist at onset. Thus, in summary, we find that there are only stable squares or rolls when $\beta \approx \delta$, that is, the coefficient of the quadratic term, $\epsilon$, is approximately zero.

Knobloch also considered the case where $\beta=\delta, \gamma \neq 0$ which he refers to as case A. In this case we find that the region of stability for the rolls and for squares is diminished to a narrow region about $\gamma=0$ as the lattice angle approaches $\frac{\pi}{6}$ or $\frac{\pi}{3}$. Since $\gamma=0$ corresponds to the degenerate hexagonal problem, again we find that squares and rolls are unstable unless $\epsilon \approx 0$.

Some time dependent computations of equation (1) have been carried out in case B by Pontes, Christov and Velarde [15]. They present results for three different sets of parameter values, two of which result in hexagons and one in which they find squares. While these results are not inconsistent with our predictions for their parameter values, two points should be born in mind when trying to make a comparison. Firstly, in [15] they use rigid rather than periodic boundary conditions in their computations, which could supress some of the instabilities we predict. Secondly, their computations are carried out at values of the bifurcation parameter which are not particularly close to critical.

\subsubsection{Stability of other planforms: the example of Marangoni convection}

As discussed in section 2, for each lattice, there are in fact six primary branches known to exist, any one of which could in principle be stable. Since many of the eigenvalues are dependent on the lattice angle $\theta_{s}$, the precise region of 
stability for each state is dependent on the choice of lattice, i.e. on the size of the domain for a box with periodic boundary conditions. Consequently, for a given physical problem, which planforms are stable at onset, can be dependent on the size of the box. We illustrate this with the example of Marangoni convection which corresponds to $\beta=-\frac{\sqrt{ } 7}{8}, \delta=-\frac{3 \sqrt{ } 7}{4}$ and $\gamma=0$. This lies within the region where squares are preferred to rolls in Knobloch's analysis. In contrast, we find that one of the following scenarios occurs:

$0<\theta_{s}<15.79^{\circ}: \quad$ Bistability of $R h_{s 2, m, n}$ and squares. e.g. $(\mathrm{m}, \mathrm{n})=(6,5)$.

$15.79<\theta_{s}<18.34^{o}: \quad R h_{s 2, m, n}$ stable. e.g. $(\mathrm{m}, \mathrm{n})=(11,8)$.

$18.34<\theta_{s}<39.26^{\circ}$ : Everything unstable, e.g. $(\mathrm{m}, \mathrm{n})=(2,1)$.

$39.26<\theta_{s}<43.71^{o}: \quad R h_{s 2, m, n}$ stable. e.g. $(\mathrm{m}, \mathrm{n})=(9,4)$.

$43.71<\theta_{s}<44.67^{\circ}: \quad$ Super squares or anti-squares are stable. e.g. $(\mathrm{m}, \mathrm{n})=(19,8)$.

$44.67<\theta_{s}<45.0^{\circ}: \quad$ Squares stable. e.g. $(\mathrm{m}, \mathrm{n})=(29,12)$.

The results for $45^{0}<\theta_{s}<90^{0}$ are essentially the same with $R h_{s 1}$ and $R h_{s 2}$ interchanged. Recall that the size of the periodic box for which these results apply is given by $\frac{\sqrt{m^{2}+n^{2}}}{k_{c}}$ and that the aspect ratio of the rhombs (rectangles) is given by $\frac{m-n}{m+n}$ for $\operatorname{Rh}_{s 1, m, n}$ and by $\frac{n}{m}$ for $\mathrm{Rh}_{s 2, m, n}$. In figure 4 we show an example of the bifurcation diagram close to onset for the case $(m, n)=(19,8)$. We know that either super squares or anti-squares are stable and have drawn the super square case. We have not calculated which of these two planforms is preferred at onset since this is determined at an $O(2(m+n)-1)=O(53)$ truncation of the bifurcation equations!

\subsection{Case II: The degenerate case $\frac{\gamma}{k_{c}^{2}}=\delta-\beta, \kappa=+1$}

In the degenerate case $\frac{\gamma}{k_{c}^{2}}=\delta-\beta$ the quadratic coefficient, $\epsilon$, is zero in equation (5) and both square and hexagonal lattices can give locally stable planforms. We have therefore evaluated the signs of the eigenvalues listed in both table 1 and table 2 which are determined at a cubic truncation of the bifurcation equations. 


\subsubsection{The stability of rolls, hexagons and squares}

Our results are presented in the form of a bifurcation set in the $(\beta, \delta)$-plane shown in figure 5 .

We first recap the relative stability results given in [1] for the subspaces $z_{4}=$ $z_{5}=z_{6}=0$ of equation (5) and $z_{3}=z_{4}=0$ of equation (4) before discussing the results of our extended analysis. First, in the subspace of the hexagonal bifurcation problem it is found:

- Hexagons are stable in region 1.

- Rolls are stable in regions 2,3 and 4.

In contrast, for the subspace of the square lattice bifurcation problem:

- Squares are stable in region 1, 2 and 3.

- Rolls are stable only in region 4 .

Together the results for the two lattices suggest that in regions 2 and 3, for an unbounded domain, that hexagons are unstable to rolls but that rolls are unstable to squares. However, there is no formal way of directly studying if hexagons are unstable to squares.

In our analysis of the finer lattices the results given above are modified. For a given hexagonal lattice we find hexagons and rhombs $\left(\mathrm{Rh}_{h 3, m, n}\right)$ are stable in region 1. Rhombs $\left(\mathrm{Rh}_{h 3, m, n}\right)$ are stable in region 2 and rolls are stable only in regions 3 and 4 . All other planforms are unstable. The position of the line dividing regions 2 and 3 is dependent on the choice of lattice. As the lattice angle approaches $\frac{\pi}{6}$, this line approaches the line $a_{1}=a_{2}$. The rhombs (rectangles) have aspect ratio depending on the lattice angle but lying between $\frac{1}{\sqrt{ } 3}$ and 1 .

For a given finer square lattice we find the stability results are unchanged for squares and rolls, however rhombs can be stable in regions 1 and 2 . In particular, for the rhombs, we find that rhombs, $R h_{s 2, m, n}$ are stable if $0<$ $\theta_{s}<\frac{\pi}{6}$ and the rhombs, $\operatorname{Rh}_{s 1, m, n}$ are stable if $\frac{\pi}{3}<\theta_{s}<\frac{\pi}{2}$. These have aspect ratio dependent on $\theta_{s}$, but again lying between $\frac{1}{\sqrt{ } 3}$ and 1 . In addition, there are 
regions of stable rhombs within region 1 which exist for $\frac{\pi}{6}<\theta<\sin ^{-1} \frac{1}{\sqrt{ } 3} \approx$ $35.3^{0}$ and for $\frac{\pi}{3}>\theta>\cos ^{-1} \frac{1}{\sqrt{ } 3} \approx 54.7^{0}$ (aspect ratios between $\frac{1}{\sqrt{ } 3}$ and $\frac{\sqrt{ } 3-1}{\sqrt{ } 2}$ ).

In summary, although the regions of stability of the hexagons and squares are unchanged by the extended bifurcation analysis, the inclusion of the rhombic states allows for bistability of hexagons and rhombs of aspect ratio close to one.

\subsubsection{Unfolding the degenerate problem}

When $\epsilon \neq 0$ the bifurcation equations for the hexagonal lattices contain a quadratic term and all planforms are necessarily locally unstable. However, if the quadratic term is sufficiently small compared with the cubic term, stable states may result through secondary bifurcations. For example, in the conventional analysis of hexagons using six critical wavevectors (figure 1(b)), for the degenerate bifurcation problem in regions 2,3 and 4 of figure 5, rolls and hexagons both bifurcate as pitchforks and rolls are stable. If a small quadratic term is added, then local to the trivial solution at $\mu_{c}$ there are no stable bifurcating branches and the hexagons bifurcate transcritically creating two distinct branches, $\mathrm{H}^{+}$and $\mathrm{H}^{-}$. An example bifurcation diagram of such a scenario is shown in figure 6(a). In this figure it can be seen that as $\mu$ is increased, there is a hysteretic transition to hexagons from the trivial solution. On further increase in $\mu$ there is a second hysteretic transition, this time between hexagons and rolls.

In our extended bifurcation analysis, again all planforms arise at pitchfork bifurcations in the degenerate case. When $\epsilon \neq 0$ both super hexagons and hexagons now bifurcate as transcritical bifurcations and all states are locally unstable. Secondary bifurcations can, however, again stabilise some of the branches. If we consider $\beta$ and $\delta$ with values corresponding to region 2 of figure 5 and if $\frac{\epsilon}{\left(1-\cos ^{2} \theta_{h}\right)}$ is sufficiently small, then the bifurcation diagram of figure 6(a) is replaced by that shown in figure 6(b). The hexagons now undergo a hysteretic transition to rhombs rather than to rolls. Remember that the aspect ratio of the rhombs is dependent on the lattice: those shown in figure $6(\mathrm{~b})$ are for the lattice $(m, n)=(3,2)$ which results in rhombs of 
aspect ratio $\frac{\sqrt{ } 3}{2} \approx 0.87$.

The criterion that $\frac{\epsilon}{\left(1-\cos ^{2} \theta_{h}\right)}$ is sufficiently small comes from requiring that the $\epsilon$ corrections to the cubic coefficients are small enough to be neglected. If this is not the case, which is inevitable for fixed $|\epsilon| \ll 1$ if the full range in $\theta_{h}$ is considered, then a sequence of more complicated transitions can occur. We illustrate this by once again considering the case of Marangoni convection.

\subsubsection{Unfolding the degenerate problem: the example of Marangoni convec- tion}

Although the Marangoni problem is nondegenerate, previous studies [13] have assumed that the quadratic term is sufficiently small and that it can legitimately be compared with the cubic terms. Specifically, the Marangoni problem has $\beta=-\sqrt{ } 7 / 8, \delta=-3 \sqrt{ } 7 / 4, \epsilon=\frac{5 \sqrt{ } 7}{8}$ and although $\epsilon$ does not appear to be small, the cubic coefficients themselves are relatively large. For example, $a_{1}=-\frac{1615}{144}$ and $a_{2}=-\frac{1707}{128}$ resulting in a saddle-node bifurcation on the hexagonal branch occurring at $\mu=-0.018$. We have calculated all the eigenvalues that are determined at cubic order for the Marangoni case for the family of hexagonal lattices and found the corresponding bifurcation lines. The results are summarised in figure 7 . The horizontal axis gives the lattice angle, $\theta_{h}$. For the eigenvalues shown, this diagram is reflection symmetric about the line $\theta_{h}=\frac{\pi}{6}$ and we have therefore only shown $0<\theta_{h}<\frac{\pi}{6}$. For simplicity, we have only shown bifurcation lines which separate stable from unstable regions of the different planforms. For $\theta_{h}$ approximately between $15^{\circ}$ and $45^{\circ}$ a transition from hexagons to rhombs occurs in a similar manner to that shown in figure $6(\mathrm{~b})$. However, for $\theta_{h}$ outside this range the $\epsilon$ corrections to the cubic coefficients result in many further secondary transitions of significance. Five main shaded regions are shown indicating different combinations of stable planforms, including regions of stable super hexagons with rhombs and/or hexagons for a cubic truncation of the bifurcation equations (note that the relative stability of super hexagons and triangles is only determined at $O(2 m-1)$ [8]). There is, in addition, a very narrow region to the left of the black region where super hexagons are the only stable planform. This region is too small to be readily discernible from figure 7 , but is apparent in the bifurcation di- 
agram shown in figure 8 . This bifurcation diagram was computed from the bifurcation equation (5) for the lattice $(m, n)=(12,7)$, i.e. $\theta_{h}=10.99^{\circ}$. Only branches which are stable for $\mu$ close to critical are drawn. In this particular case there is a region of bistability between hexagons and super hexagons. A hysteretic transition between hexagons and super hexagons can occur. An illustration of the super hexagon state for the case $(m, n)=(12,7)$ is also shown. Although such complex patterns have not yet been seen in convection problems, recent experimental results of the Faraday crispation experiments do show such "superlattice patterns" [7].

Note that, if all lattices (all $\theta_{h}$ ) are considered, then hexagons are necessarily unstable ( see $0<\theta_{h}<8^{\circ}$ in figure 7 ). However, in this case where we have fixed $\epsilon \neq 0$, it is unclear whether this local problem is still valid for the amplitudes where secondary bifurcations arise. Nevertheless, it demonstrates some of the intriguing possibilities associated with this bifurcation problem.

\section{Conclusions}

Standard low-dimensional bifurcation analyses of squares and hexagons give only a restricted stability analysis of these planforms. We have shown that, with one additional perturbation calculation, i.e. the calculation of $C_{R h}$, all the additional coefficients required to apply the extended stability analysis of [11] are determined. We have performed this calculation for the case of the long-wavelength convection equation (1) and analysed the results in two main cases. In Case I we found that extending the stability results significantly increased the known region of instability for squares in an unbounded domain. In particular, we find that squares are only stable at onset if $\epsilon \approx 0$. This is interesting given that it is already known that all solutions which are periodic on a hexagonal lattice are unstable at onset unless the degeneracy condition, $\epsilon=0$, is met. For a given box with periodic boundary conditions we found that the predicted planform at onset was strongly dependent on the size of the box: some of the more exotic planforms such as super squares and anti-squares could be stable. In Case II we showed that regions of bistability of rhombs and hexagons exist. This gave a formal setting for studying the transition between hexagons and rhombs of aspect ratio close to 1 (although not squares). 


\section{Acknowledgement}

The research of MS was supported by NSF grant DMS-9404266 and by an NSF CAREER award DMS-9502266. ACS thanks the Nuffield Foundation and the Royal Society for their support. The authors also appreciate the hospitality of the Hat Creek Radio Observatory, where much of the work for this paper was done. 


\section{References}

[1] E. Knobloch. Pattern selection in long-wavelength convection. Physica D $4 \mathbf{1}$ (1990) 450-479.

[2] B.A. Malomed, A.A. Nepomnyashchii and M.I. Tribelskii. Two-dimensional quasiperiodic structures in nonequilibrium systems. Sov. Phys. JETP 69 (1989) 388-396.

[3] J.W. Swift. Bifurcation and symmetry in convection PHD Thesis (1984) University of California, Berkeley.

[4] E. Buzano and M. Golubitsky. Bifurcation on the hexagonal lattice and the planar Bénard problem. Philos. Trans. R. Soc. A 308 (1983) 617-667.

[5] M. Golubitsky, E. Knobloch and J.W. Swift. Symmetries and pattern selection in Rayleigh-Bénard convection. Physica D 10 (1984) 249-276.

[6] K. Brattkus and S.H. Davis. Cellular growth near absolute stability Phys. Rev. $B 38$ (1988) 11452-60.

[7] A. Kudrolli, B. Pier and J.P. Gollub. Superlattice patterns in surface waves. Physica D To appear (1998).

[8] M. Silber and M.R.E. Proctor. Nonlinear competition between small and large hexagonal patterns. preprint (1997).

[9] B. Dionne. Spatially Periodic Patterns in Two and Three Dimensions. Ph.D. Thesis, University of Houston (1990).

[10] B. Dionne and M. Golubitsky. Planforms in two and three dimensions. $Z$. Angew. Math. Phys. 43 (1992) 36-62.

[11] B. Dionne, M. Silber and A.C. Skeldon. Stability results for steady, spatiallyperiodic planforms. Nonlinearity 10 (1997) 321-353.

[12] M.R.E. Proctor. Planform selection by finite-amplitude thermal convection between poorly conducting slabs. J. Fluid Mech. (1981) 469-485.

[13] L. Shtilman and B. Sivashinsky. Hexagonal structure of large scale Marangoni convection. Physica D 52 (1991) 472-488.

[14] E. Ihrig and M. Golubitsky. Pattern selection with $\mathrm{O}(3)$ symmetry. Physica $D$ 12 (1984) 1-33.

[15] J. Pontes, C.I. Christov and M.G. Velarde Numerical study of patterns and their evolution in finite geometries. Int. J. of Bif. and Chaos 6 (1996) 1883-1890. 
(a)

$\bullet$

$\bullet$

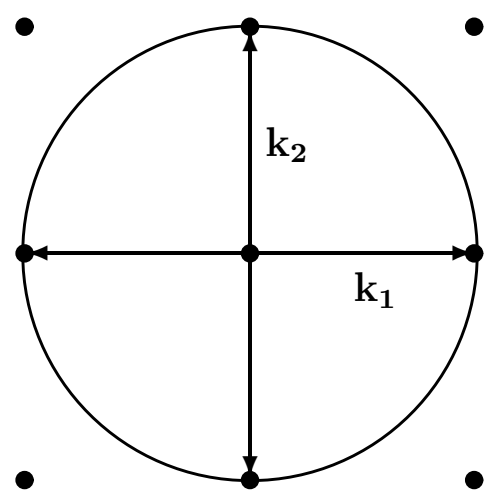

$\bullet$

-

(2)

$\bullet$

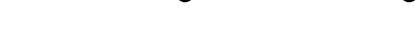

-

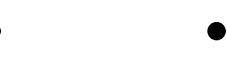

$\bullet$

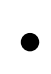

$\bullet$

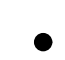

(b)
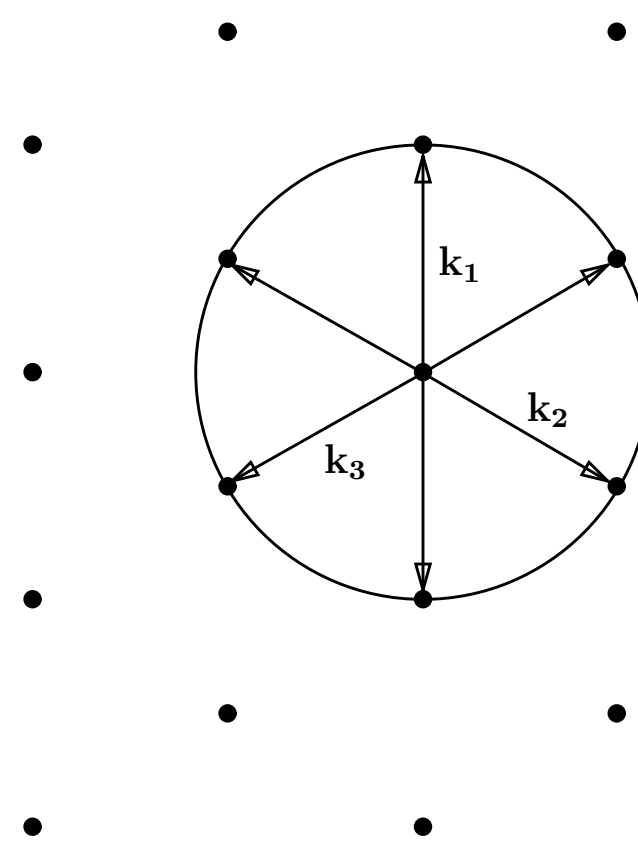

Fig. 1. (a) Square lattice generated by four wavevectors on the critical circle oriented at $\frac{\pi}{2}$ to each other. (b) Hexagonal lattice generated by six wavevectors on the critical circle oriented at $\frac{\pi}{3}$ to each other. 
(a)

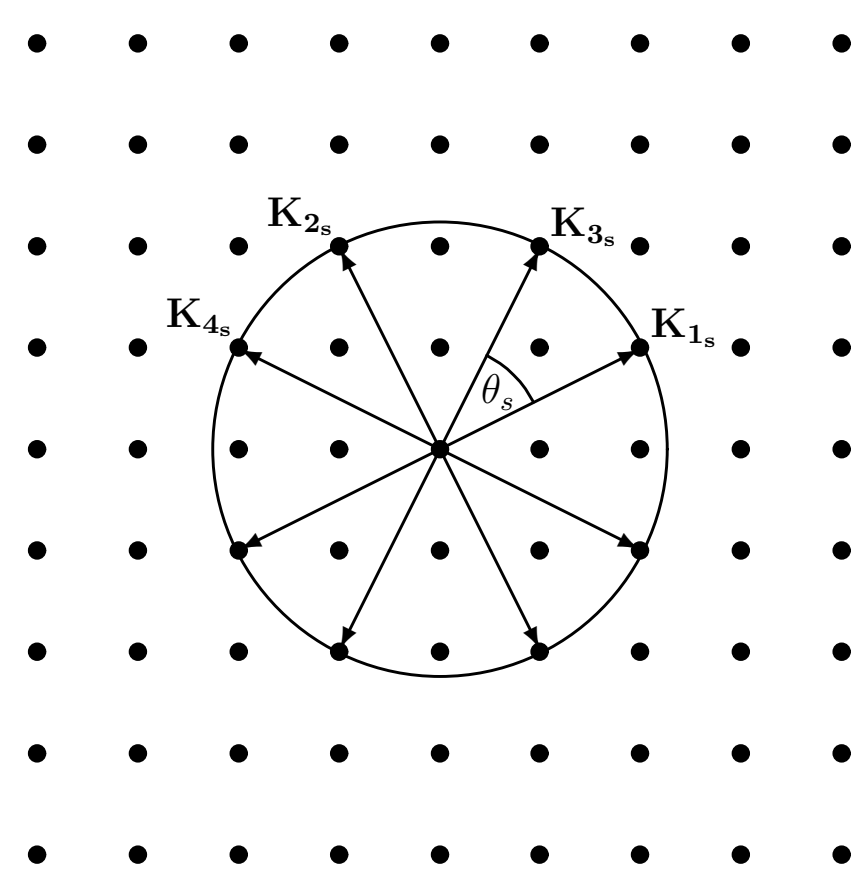

(b)

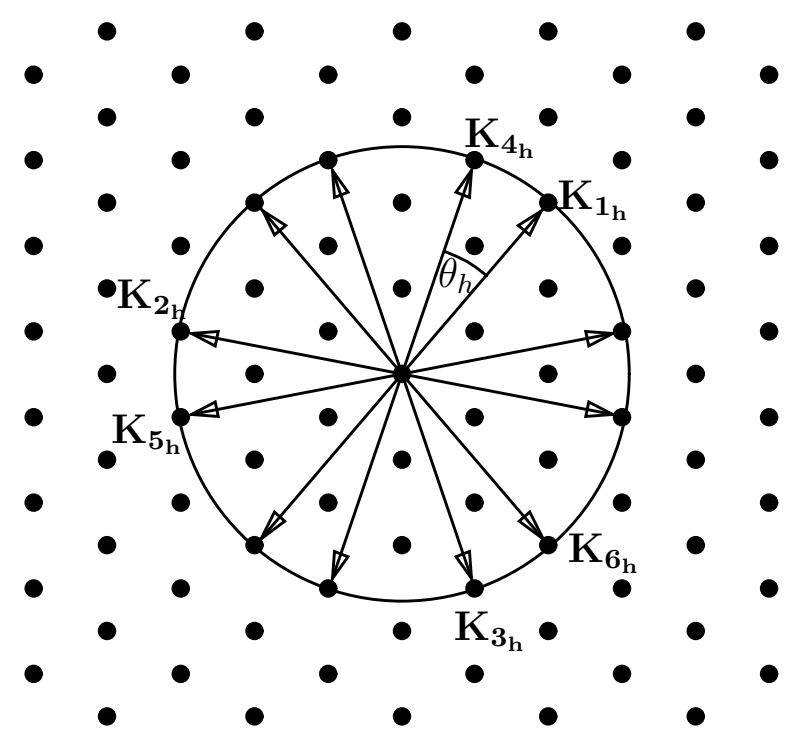

Fig. 2. (a) Square lattice generated by eight wavevectors on the critical circle. This case shows $(m, n)=(2,1)$. (b) Hexagonal lattice generated by twelve wavevectors on the critical circle. This case shows $(m, n)=(3,2)$. 

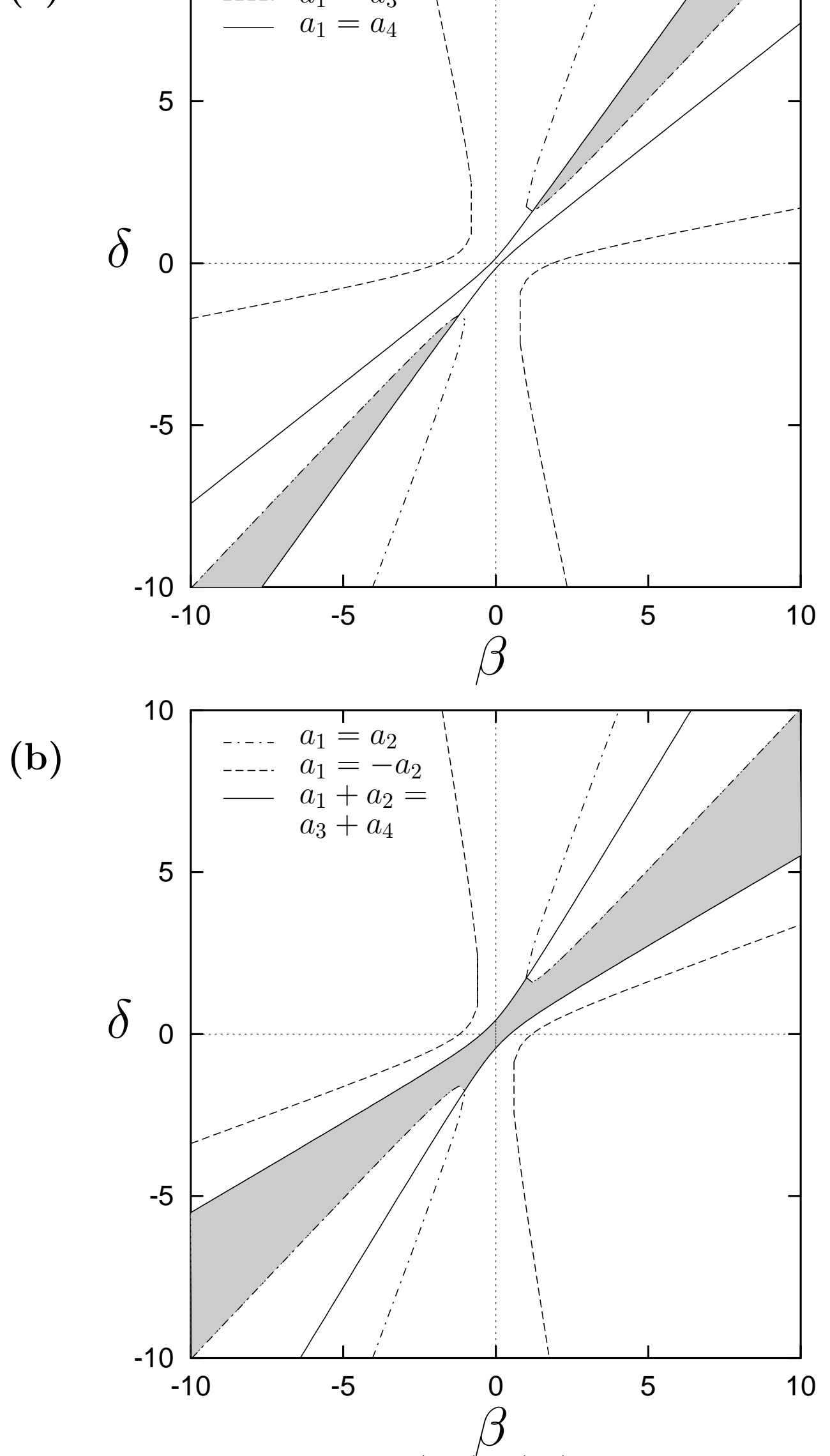

Fig. 3. Bifurcation sets in case I for $(m, n)=(2,1)$. The lines indicate transitions in the number of negative eigenvalues. (a) Bifurcation set for rolls. The shaded region shows the region where rolls are preferred. (b) Bifurcation set for squares. The shaded region shows the region where squares are preferred. 


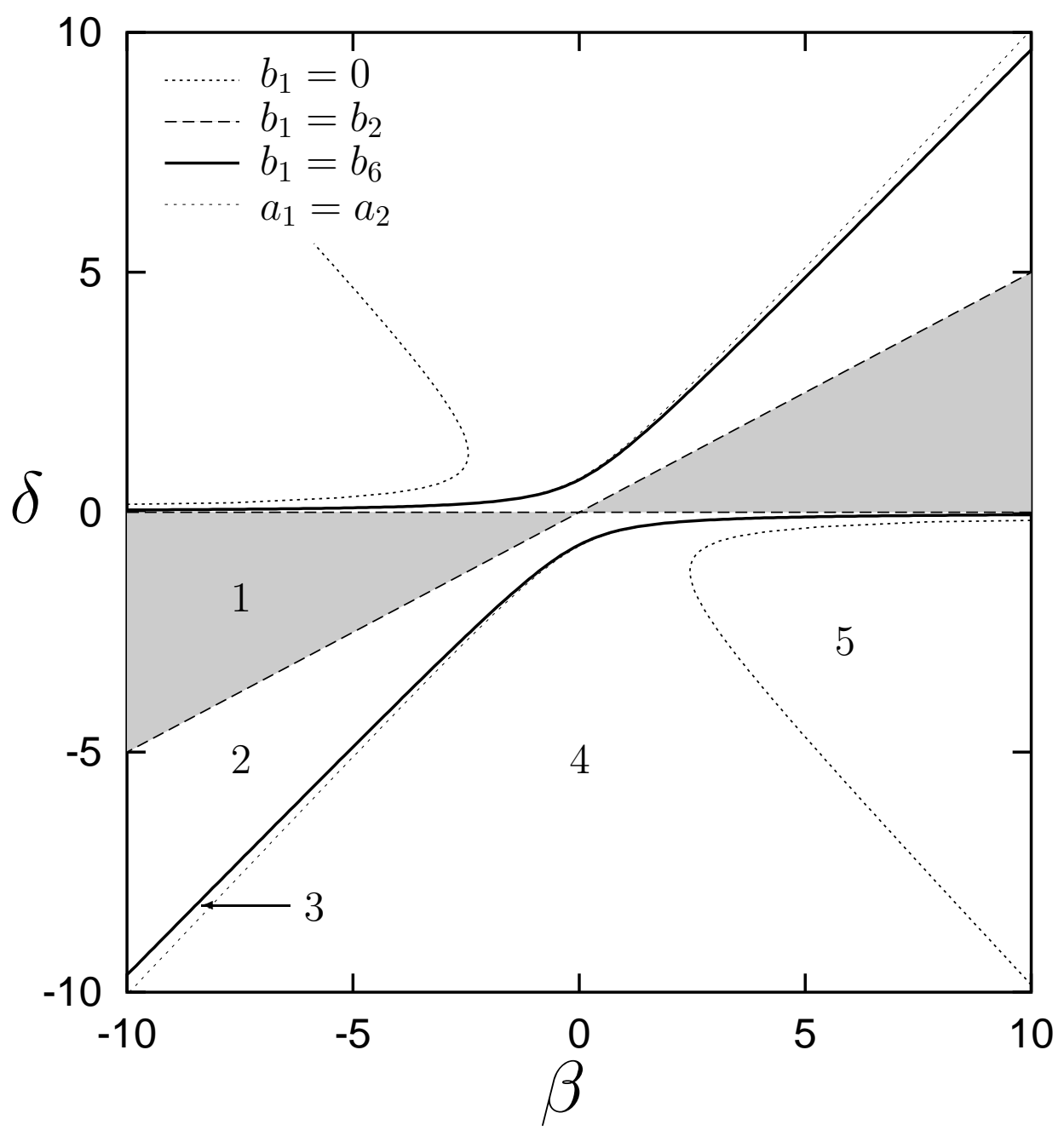

Fig. 5. Bifurcation set in the case $\epsilon=0$. Only critical eigenvalues which divide stable from unstable planforms are shown. The different regions are discussed in the text. 


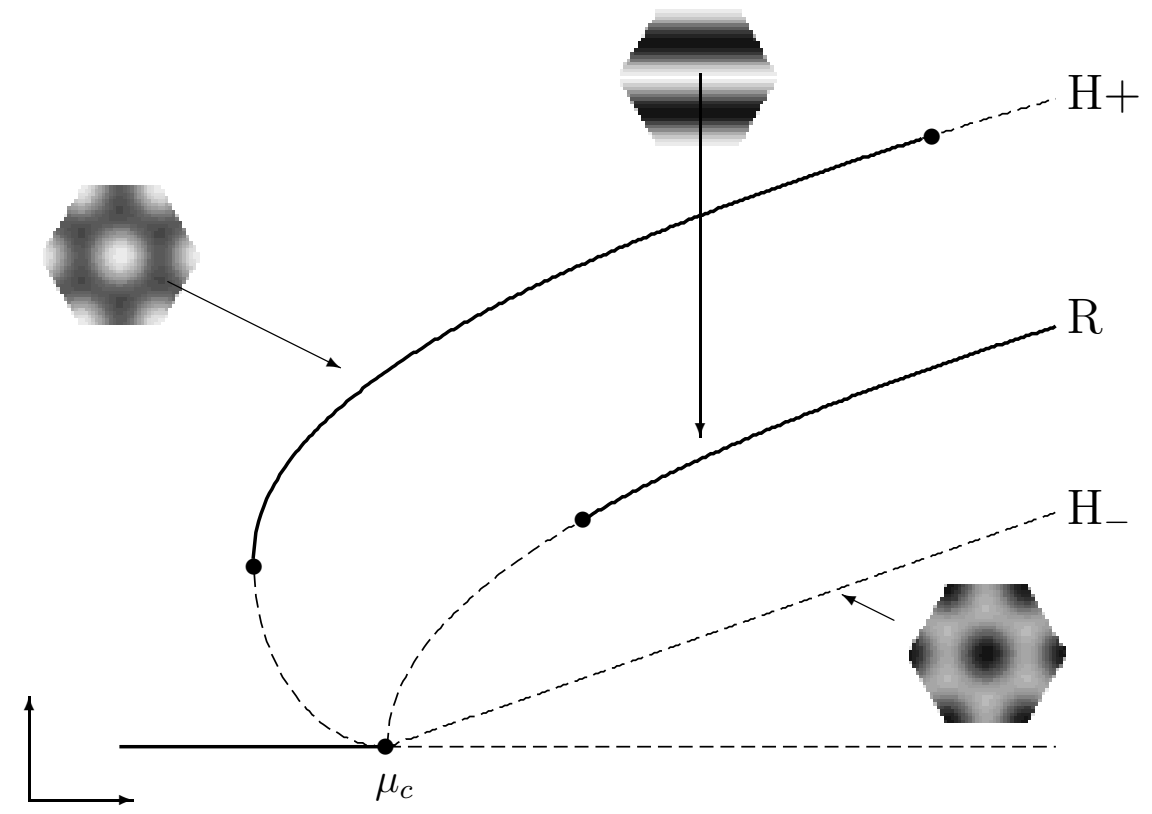

(b)

\section{$\mu$}

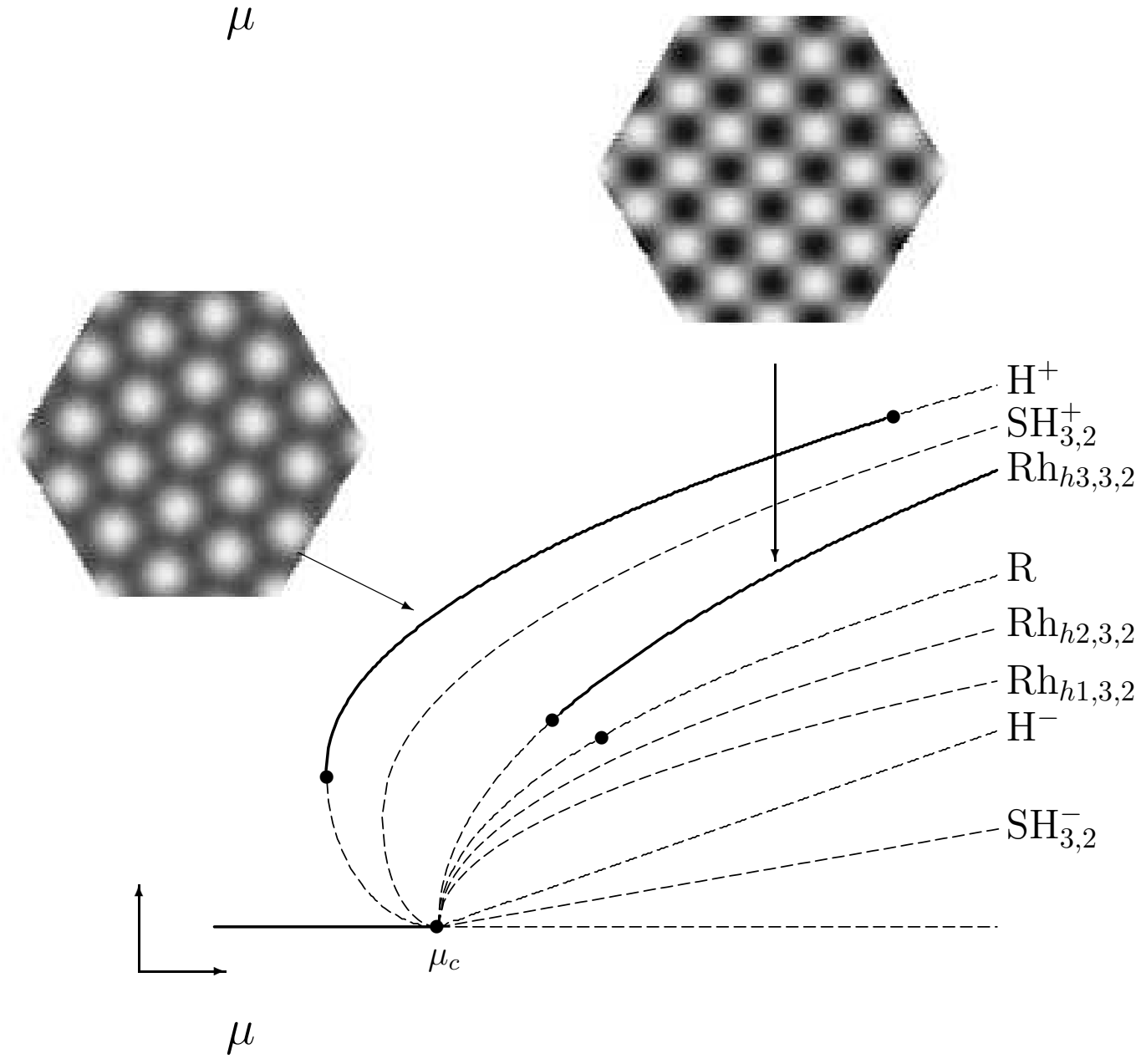

Fig. 6. Unfoldings of the bifurcation diagrams relevant to region 2 in figure 5 for $\epsilon$ sufficiently small. (a) The bifurcation diagram obtained by considering the subspace $z_{4}=z_{5}=z_{6}=0$ of equation (5). (b) The new bifurcation diagram when the additional branches in our extended analysis is considered. Note that the rhombic (rectangular) pattern has an aspect ratio close to 1. 


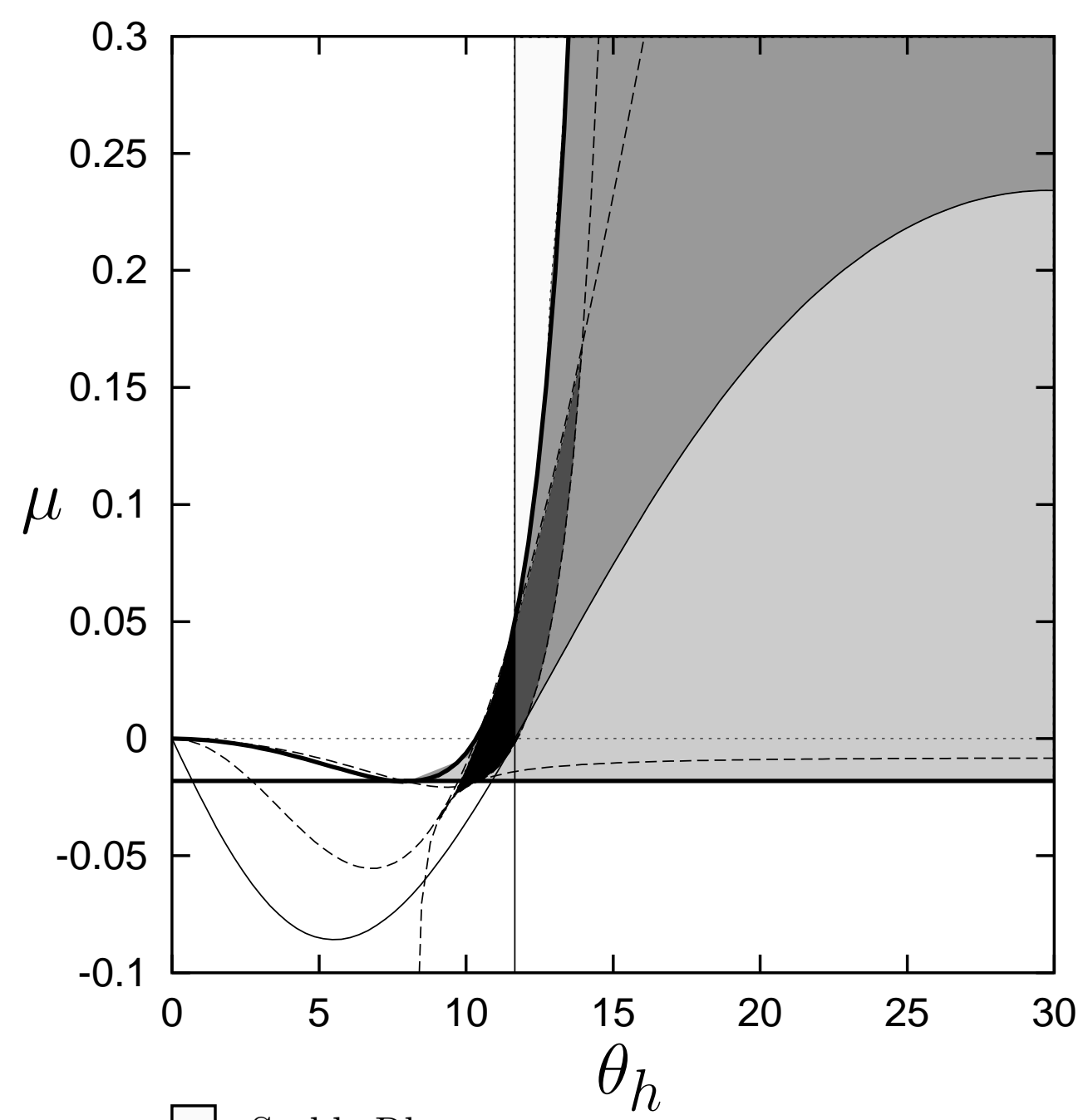

$\square$ Stable $\operatorname{Rh}_{h 3, m, n}$

$\square$ Stable hexagons

$\square$ Stable hexagons and $\mathrm{Rh}_{h 3, m, n}$

$\square$ Stable hexagons, $\mathrm{Rh}_{h 3, m, n}$ and super hexagons

Stable hexagons and super hexagons

- Bifurcation lines for hexagons

— Bifurcation lines for $\operatorname{Rh}_{h 3, m, n}$

---- Bifurcation lines for super hexagons

Fig. 7. Bifurcation set for the Marangoni problem, $\beta=-\frac{\sqrt{ } 7}{8}, \delta=-\frac{3 \sqrt{ } 7}{4}, \epsilon=\frac{5 \sqrt{ } 7}{8}$ as a function of the lattice angle $\theta_{h}$. 


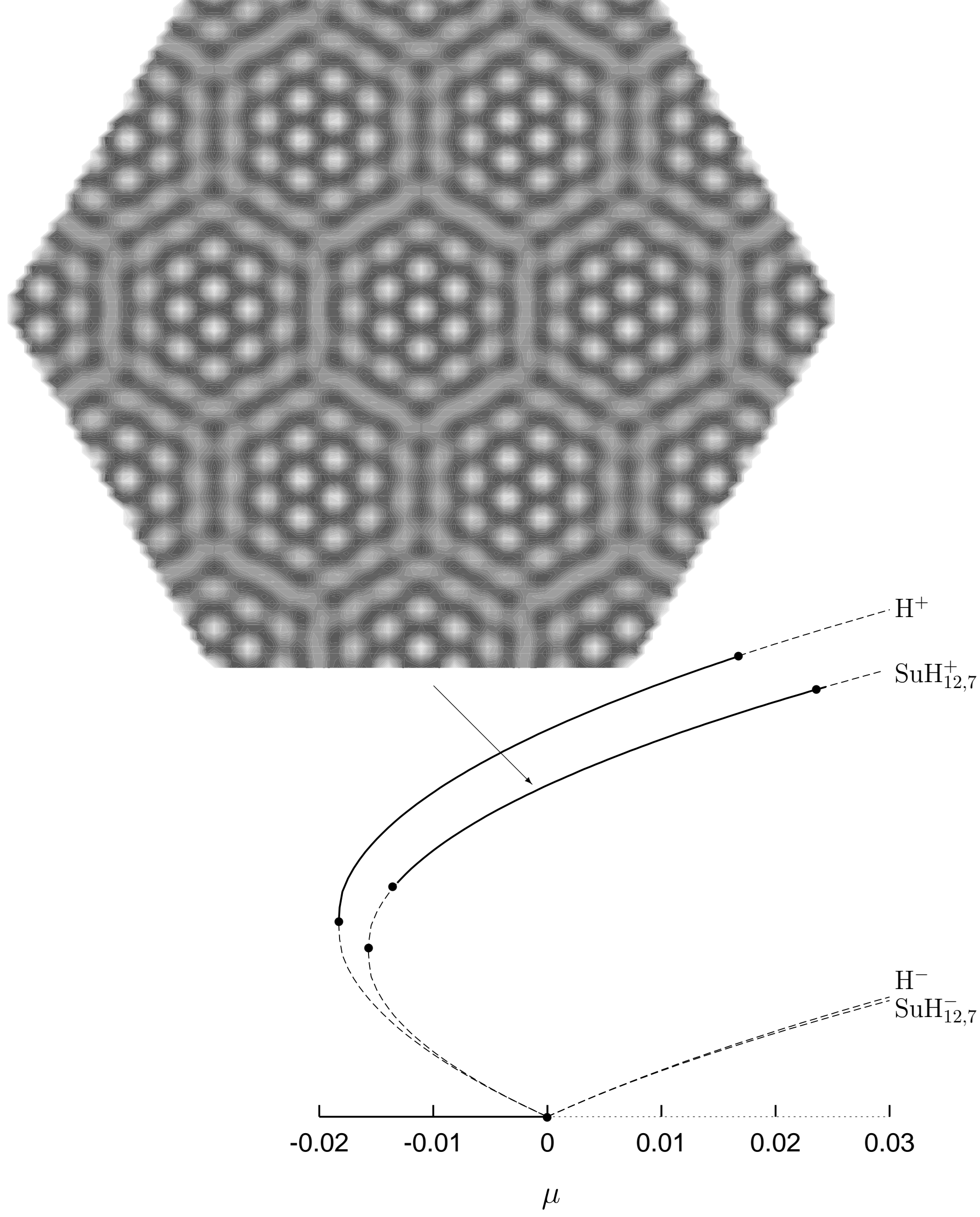

Fig. 8. Example bifurcation diagram for the Marangoni problem in a box with periodic boundary conditions. This case is for $(m, n)=(12,7)$. Only branches which can be stable are shown. 